Article

\title{
A Novel Thin-Film Nanocomposite Nanofiltration Membrane by Incorporating 3D Hyperbranched Polymer Functionalized 2D Graphene Oxide
}

\author{
Quanling Xie ${ }^{1,3, *} \mathbb{D}$, Shishen Zhang ${ }^{1,2}$, Hanjun Ma ${ }^{1,2}$, Wenyao Shao ${ }^{2, *}$, Xiao Gong ${ }^{2}$ and \\ Zhuan Hong $1,3, *$ \\ 1 Engineering Research Center of Marine Biological Resource Comprehensive Utilization, SOA, \\ The Third Institute of Oceanography of the State Oceanic Administration, Xiamen 361005, China; \\ 20620151152271@stu.xmu.edu.cn (S.Z.); 20620171151152@stu.xmu.edu.cn (H.M.) \\ 2 Department of Chemical and Biochemical Engineering, College of Chemistry and Chemical Engineering, \\ Xiamen University, Xiamen 361005, China; 20420162201530@stu.xmu.edu.cn \\ 3 Fujian Collaborative Innovation Center for Exploitation and Utilization of Marine Biological Resources, \\ Xiamen 361005, China \\ * Correspondence: qlxie@tio.org.cn (Q.X.); wyshao@xmu.edu.cn (W.S.); zhong@tio.org.cn (Z.H.)
}

Received: 22 October 2018; Accepted: 8 November 2018; Published: 12 November 2018

\begin{abstract}
In order to develop a high-performance thin-film nanocomposite (TFN) nanofiltration (NF) membrane, the functionalized graphene-based nanomaterial (GO-HBE-COOH) was synthesized by combining two-dimensional graphene oxide (GO) with a three-dimensional hyperbranched polymer, which was used as the novel nanofiller and successfully embedded into the polypiperazine-amide (PPA) active layers on polysulfone (PSU) substrates via interfacial polymerization (IP) process. The resultant NF membranes were characterized using ATR-FTIR, SEM, and AFM, while their performance was evaluated in terms of water flux, salt rejection, antifouling ability, and chlorine resistance. The influence of GO-HBE-COOH concentration on the morphologies, properties, and performance of TFN NF membranes was investigated. With the addition of $60 \mathrm{ppm}$ GO-HBE-COOH, the TFN-GHC-60 NF membrane exhibited the optimal water flux without a sacrifice of the salt rejection. It was found that the introduction of GO-HBE-COOH nanosheets favored the formation of a thinner and smoother nanocomposite active layer with an enhanced hydrophilicity and negative charge. As a result, TFN NF membranes demonstrated a superior permeaselectivity, antifouling ability, and chlorine resistance over the conventional PPA thin-film composite (TFC) membranes.
\end{abstract}

Keywords: nanofiltration; thin-film nanocomposite; graphene oxide; hyperbranched polyester; carboxylation

\section{Introduction}

Pressure-driven membrane separation technology with a selectively permeable membrane belongs to a kind of physical separation process, which has the advantages of high efficiency, energy saving ability, modular design, friendliness to the environment, and so on. Nanofiltration (NF) is the newest of the four classes of pressure-driven membranes whose pore size is between reverse osmosis (RO) and ultrafiltration (UF). To date, polyamide (PA) thin-film composite (TFC) NF is widely applied in many industries including water treatment, pharmaceutical and biotechnology, and food engineering due to its distinguishing characteristics such as low rejection to monovalent ions, high rejections to divalent ions and low Mw compounds, higher flux, and lower operation pressure [1]. Nevertheless, PA-TFC NF membrane still encounters great challenges such as a trade-off between permeability and selectivity, 
membrane fouling, chlorination, and concentration polarization [2]. In order to overcome these challenges, organic-inorganic hybrid membranes have been developed by combining the advantages of organic materials and inorganic materials. In recent years, the rapid development of nanomaterials plays an important role in promoting the development of novel composite membranes. Especially, the application of two-dimensional (2D) graphene nanomaterials, represented by graphene oxide (GO), has received increasing attention. Thin-film nanocomposite (TFN) NF membranes incorporating an appropriate amount of GO nanosheets into the top active layer and/or the porous support layer have demonstrated a superior performance over the traditional PA-TFC NF membranes [3-10]. Because the top PA active layer is believed to play a crucial role in the permeaselectivity and antifouling performance, most studies related to GO-modified TFN NF membranes focus on the optimization of the active layer rather than the porous support layer.

GO with abundant oxygen-containing functional groups is normally regarded as a kind of hydrophilic nanomaterial. Actually, the structure of GO has been clarified to be amphipathic with an edge-to-center distribution of hydrophilic and hydrophobic domains [11-13]. Therefore, the chemical functionalization of GO is needed to enhance the hydrophilicity, dispersibility as well as the compatibility with the polymer matrices. Our group used a small molecular modifier, maleic anhydride (MAH), reacted with the electrophilic groups of GO and synthesized MAH functionalized GO (MAH-GO) [14]. It was found that TFN NF membranes introducing MAH-GO into the active layer displayed a better performance than GO-embedded TFN NF membranes.

Hyperbranched polymers (HBPs) have attracted more and more interest in membrane preparation due to the three-dimensional (3D) spherical structure and unique physicochemical properties compared to their linear analogues [15-17]. Moreover, HBPs showed a good interfacial compatibility with cross-linked polyamide matrix and the intra-molecules nanovoids inside HBPs offered additional water channels to enhance membrane flux. The modified HBPs containing amino, alcohol, and carboxylic acid end groups were used for reducing protein adsorption by introducing a high density of hydrophilic functional groups at the membrane surface [18]. Many kinds of HBPs such as hyperbranched polyester (HPE) [17,19-23] and hyperbranched polyethyleneimine [24-27] have been explored to improve membrane performance. Kong et al. [20] introduced a small amount of HPE used as the macromonomer into the aqueous phase. They found that the HPE embedded in the active layer significantly increased the permeability without sacrificing the salt rejection for the prepared TFC NF membranes. Furthermore, HPE with a 3D spherical structure preferred to be deposited on the top surface of the substrate rather than snake through the pores like linear molecules [17].

In order to make use of 2D graphene-based nanomaterials and 3D HBPs in this study, GO was firstly modified with hydroxyl-terminated HPE to synthesize HPE functionalized GO (GO-HPE), and then GO-HPE was further reacted with succinic anhydride to obtain GO-HPE-COOH containing a large number of hydrophilic carboxyl groups. Finally, the resultant GO-HPE-COOH nanosheets used as novel nanofillers were added into the aqueous phase and incorporated into the polypiperazine-amide (PPA) active layer during the interfacial polymerization (IP) process. The effect of GO-HPE-COOH concentration on the morphologies, properties, and performance of the resulting TFN NF membranes were investigated in-depth. Furthermore, the influencing mechanisms of GO-HPE-COOH on the IP process and the formation of the nanocomposite active layer were discussed.

\section{Experimental}

\subsection{Materials}

Polysulfone (PSU, Ultrason ${ }^{\circledR S}$ 6010), PVP K30, and PVP K90 were produced by BASF (Ludwigshafen, Germany). Succinic anhydride, tetrahydrofuran (THF), dimethylformamide (DMF), dimethyl sulfoxide (DMSO), triethylamine (TEA), n-hexane, sodium hypochlorite, sodium sulfate, magnesium sulfate, sodium chloride, magnesium chloride, and hydrochloric acid (36-38\%) were of analytical grade and produced by Sinopharm (Shanghai, China). $\mathrm{SOCl}_{2}$, piperazine (PIP), 
and trimesoyl chloride (TMC) were purchased from Aladdin (Shanghai, China). HBE was produced by HyPerBranched Polymers Co. Ltd (Wuhan, China). Bovine serum albumin (BSA) was purchased from BBI Life Sciences Corporation (Shanghai, China).

\subsection{Preparation of $\mathrm{GO}$ and $\mathrm{GO}-\mathrm{HBE}-\mathrm{COOH}$}

GO were obtained using the modified Hummer's method [14]. The esterification procedure for $\mathrm{GO}$ was as follows (Figure 1): $100 \mathrm{mg}$ of GO were reacted in $20 \mathrm{~mL}$ of $\mathrm{SOCl}_{2}$ (containing $1 \mathrm{~mL} \mathrm{DMF}$ ) at $70{ }^{\circ} \mathrm{C}$ for $24 \mathrm{~h}$ to convert the carboxylic acids into acyl chlorides (acyl chloride-derivative GO, GOCl). After centrifugation at $12,000 \mathrm{rpm}$ for $10 \mathrm{~min}$, the supernatant was decanted and the remaining solid was washed three times with anhydrous THF. The obtained GOCl solid was dried at room temperature under vacuum. A mixture of the resulting $\mathrm{GOCl}$ and $4.5 \mathrm{~g} \mathrm{HBE}(45 \mathrm{mmol}$ equivalent to the $\mathrm{OH}$ group) was dispersed in $100 \mathrm{~mL}$ DMSO and stirred vigorously at $50{ }^{\circ} \mathrm{C}$ for $65 \mathrm{~h}$. After centrifugation, the remaining solid was washed three times with methanol. The obtained GO-HBE solid was dried at room temperature under vacuum. Then the carboxylation procedure for GO-HBE was carried out as follows: the obtained GO-HBE were dispersed in $60 \mathrm{~mL}$ THF. A total of $2.07 \mathrm{~g}$ of succinic anhydride and $1.0 \mathrm{~mL}$ of TEA were dissolved in $60 \mathrm{~mL}$ THF, which was mixed with GO-HBE suspension and condensate refluxed at $70{ }^{\circ} \mathrm{C}$ for $20 \mathrm{~h}$. After centrifugation, the remaining solid was washed three times with THF. The obtained GO-HBE-COOH solid was dried at room temperature under vacuum.

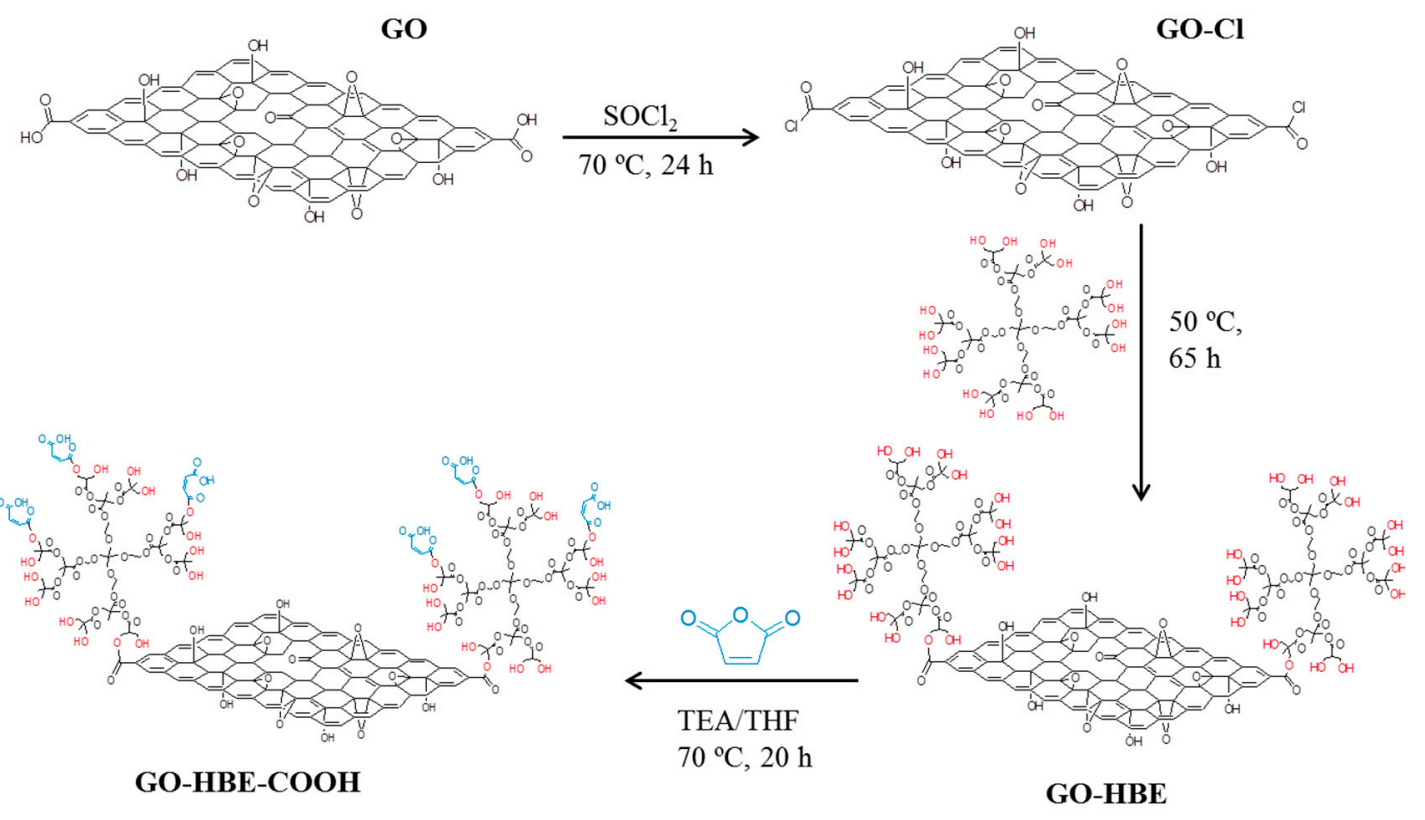

Figure 1. The schematic of the synthesis route of GO-HBE-COOH.

\subsection{Preparation of Composite NF Membrane}

The structure and property of the porous support layer are also in close relation to the performance of the resultant composite NF membrane. During the preliminary study in our laboratory, the optimum PSU concentration and casting solvent for fabricating the porous supports was found to be $15.0 \mathrm{wt} . \%$ and NMP, which was consistent with the previous report [28]. GO-HBE-COOH nanofillers with various concentrations (0-80 ppm) was first dispersed into the $2.0 \mathrm{wt} . \%$ PIP aqueous solution containing $2.0 \mathrm{wt} . \%$ TEA as an acid acceptor by ultrasonic treatment for $40 \mathrm{~min}$. Then $100 \mathrm{~mL}$ of the resultant aqueous solution was poured on the top of the PSU support and held for $2 \mathrm{~min}$ before removing the excess aqueous solution. Subsequently, $100 \mathrm{~mL}$ of an organic solution containing $0.1 w / v \%$ TMC dissolved in n-hexane was poured on the top of the PIP saturated PSU support and drained off after 1 min contact time. Finally, the resulting composite membranes were heat cured at $60^{\circ} \mathrm{C}$ for 8 min to enhance the IP reaction. The obtained nanocomposite membranes were named TFN-GHC-X, 
where $\mathrm{X}$ denoted the GO-HBE-COOH concentration (ppm) in the aqueous solution. The composite NF membrane without GO-HBE-COOH was named TFC-blank.

\subsection{Characterization of $\mathrm{GO}$ and $\mathrm{GO}-\mathrm{HBE}-\mathrm{COOH}$}

The functional groups and chemical structure differences of GO and GO-HBE-COOH were characterized by Fourier transform infrared spectroscopy (FT-IR, Bruker VERTEX 70, Fällanden, Switzerland). Their morphologies were observed with a transmission electron microscopy (TEM, TALOS F200, Hillsboro, OR, USA) operated at $200 \mathrm{kV}$. The X-ray diffraction (XRD) with Cu $\mathrm{K} \alpha$ radiation $(\lambda=0.154 \mathrm{~nm}, \mathrm{D} / \mathrm{max}-\mathrm{rB} 12 \mathrm{~kW}$, Rigaku, Tokyo, Japan) was performed (with an emission current of $15 \mathrm{~mA}$ and the accelerating voltage of $35 \mathrm{kV}$ ) and the XRD data were processed by the MDI Jade 6.0 software.

\subsection{Characterization of Composite NF Membranes}

Attenuated total reflectance-Fourier transform infrared spectroscopy (ATR-FTIR, Bruker VERTEX 70, Fällanden, Switzerland) was employed to characterize the presence of GO-HBE-COOH in the fabricated TFN membranes. The membrane hydrophilicity was assessed by the static water contact angle using a contact angle goniometer (SPCAX3, HARKE, Beijing, China). The instantaneous contact angle was recorded after $30 \mathrm{~s}$ of equilibration time and at least five water contact angles were averaged to get a reliable value. The top membrane surfaces and their cross-sectional morphologies were observed using a scanning electron microscope (SEM, Sigma, Zeiss, Jena, Germany) operating at $15 \mathrm{kV}$. An atomic force microscope (AFM, MI5500, Agilent, Santa Clara, CA, USA) was used to characterize the morphology and roughness of the top membrane surface in tapping mode using the probe (OLTESPA-R3, Bruker, Billerica, MA, USA) with a spring constant of $2 \mathrm{~N} \cdot \mathrm{m}^{-1}$.

\subsection{Membrane Performance}

The NF filtration experiments were performed using a laboratory membrane separation device (FlowMem-0021-HP, Xiamen Filter and Membrane Technology, Xiamen, China). The composite membranes were pre-pressurized at $0.6 \mathrm{MPa}$ and $25 \pm 1^{\circ} \mathrm{C}$ for $30 \mathrm{~min}$ before measuring their separation performance. Four representative salt solutions were adopted to evaluate the retention and selectivity. The diffusion coefficient and Stokes radius of various ions are listed in Table 1 [29]. The water flux and the rejection $(R)$ were calculated using the Equations (1) and (2), respectively:

$$
J=\frac{V}{A \Delta t}
$$

where $J$ is the water flux $\left(\mathrm{L} \cdot \mathrm{m}^{-2} \cdot \mathrm{h}^{-1}\right), V$ is the permeate volume $(\mathrm{L}), A$ is the effective membrane area $\left(\mathrm{m}^{2}\right)$, and $\Delta t$ is the filtration time $(\mathrm{h})$.

$$
R(\%)=\left(1-\frac{C_{p}}{C_{c}}\right) \times 100 \%
$$

where $C_{p}$ and $C_{c}$ refer to the salt concentration in the permeate and in the concentrate, respectively.

Table 1. The parameters of relevant ions.

\begin{tabular}{cccc}
\hline Ions & $z$ & $\boldsymbol{D ( \mathbf { 1 0 } ^ { - \mathbf { 3 } } \mathbf { m m } ^ { \mathbf { 2 } } \cdot \mathbf { s } ^ { - \mathbf { 1 } } )}$ & $\boldsymbol{r}_{\mathbf{s}}(\mathbf{n m})$ \\
\hline $\mathrm{Na}^{+}$ & 1 & 1.333 & 0.183 \\
$\mathrm{Mg}^{+}$ & 2 & 0.706 & 0.345 \\
$\mathrm{Cl}^{-}$ & -1 & 2.032 & 0.120 \\
$\mathrm{SO}_{4}{ }^{2-}$ & -2 & 1.065 & 0.229 \\
\hline
\end{tabular}

Note: $z, D$ and $r_{\mathrm{s}}$ refer to the charge number, diffusion coefficient and Stokes radius. 
The antifouling abilities of the TFC-blank and the selected TFN membranes were assessed using BSA as the model foulant. First, the operation of water filtration was running for $60 \mathrm{~min}$ at $0.6 \mathrm{MPa}$. Then the operation of the BSA filtration was carried out for another $60 \mathrm{~min}$ using a $2 \mathrm{~g} \cdot \mathrm{L}^{-1} \mathrm{BSA}$ solution instead of pure water. After washing the fouled membranes with pure water, a complete cycle including water filtration and BSA filtration were repeated.

The chloride resistance of the TFC-blank and the selected TFN membranes was evaluated based on the variation of permeaselectivity. The tested membranes were immersed in a $2000 \mathrm{ppm} \mathrm{NaClO}$ solution with a neutral $\mathrm{pH}$. In order to remove the residual $\mathrm{NaClO}$ solution before reassessing the membrane performance, the chlorinated membrane was rinsed thoroughly with pure water and soaked in pure water for at least $1 \mathrm{~h}$.

\section{Results and Discussion}

\subsection{Characterizations of $\mathrm{GO}$ and $\mathrm{GO}-\mathrm{HBE}-\mathrm{COOH}$}

The functional groups in GO and GO-HBE-COOH were analyzed via FT-IR (Figure 2). As reported in our previous studies [14,30], the GO spectrum displayed several typical characteristic peaks assigned to the oxygen-containing hydrophilic groups on the basal plane and edge: the broad band at $3390 \mathrm{~cm}^{-1}$ and the peak at $1365 \mathrm{~cm}^{-1}$ were assigned to the stretching vibration and deformation vibration of $-\mathrm{OH}$; the peak at $1732 \mathrm{~cm}^{-1}$ was assigned to the $\mathrm{C}=\mathrm{O}$ stretching vibration of carboxylic groups; two bands at 1230 and $1090 \mathrm{~cm}^{-1}$ were assigned to the $\mathrm{C}-\mathrm{O}$ in epoxy groups and $\mathrm{C}-\mathrm{O}$ in alkoxy groups, respectively. After the surface covalent modification with HBE and succinic anhydride, the broad band at $3390 \mathrm{~cm}^{-1}$ became sharp, and two new peaks appearing at $2938 \mathrm{~cm}^{-1}$ and $2852 \mathrm{~cm}^{-1}$ were assigned to the $\mathrm{C}-\mathrm{H}$ stretching vibration from methyl and methylene. Furthermore, the peak intensity at $1732 \mathrm{~cm}^{-1}$ assigned to the $\mathrm{C}=\mathrm{O}$ stretching vibration of carboxylic groups was significantly enhanced. All these above observations confirmed the successful synthesis of GO-HBE-COOH.

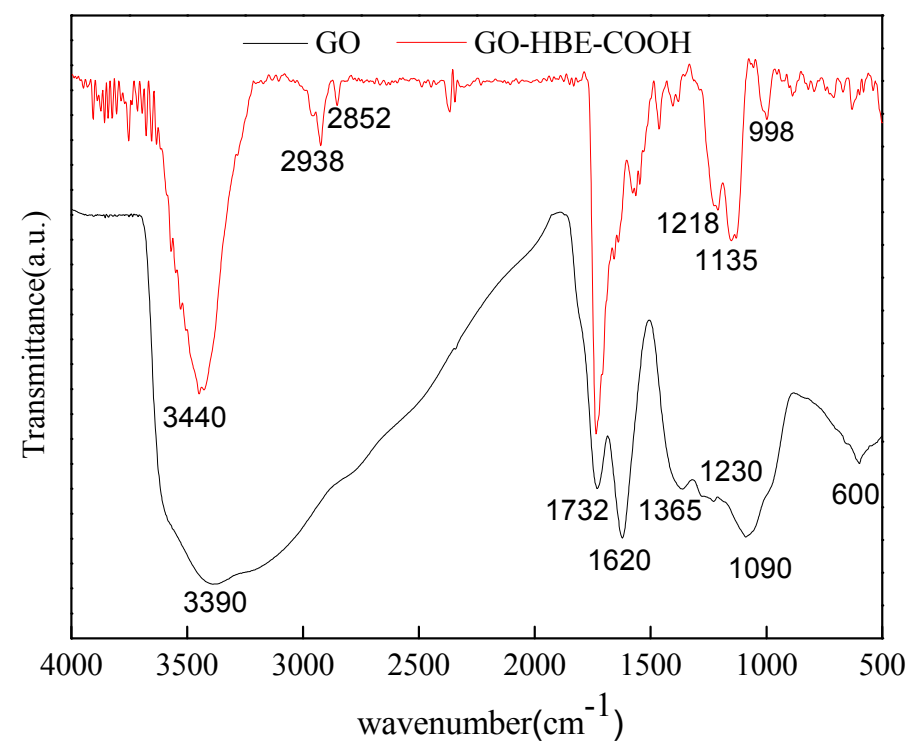

Figure 2. The FT-IR spectra of GO and GO-HBE-COOH.

According to Figure 3, both GO and GO-HBE-COOH displayed one to several layers with some wrinkling within large flat areas, leading to the color being varied from transparent to semitransparent. Obviously, GO-HBE-COOH presented a similar lamellar structure and morphology as GO nanosheets. It was deduced that GO-HBE-COOH attaching hyperbranched polymer via covalent bonding did not alter the original structure and morphology of the GO nanosheets.

The XRD patterns of GO and GO-HBE-COOH are shown in Figure 4. A sharp feature diffraction peak at $10.08^{\circ}$ was attributed to the (002) plane of GO [31], which corresponded to a $0.877 \mathrm{~nm}$ d-space 
calculated from the Bragg equation. After introducing the hyperbranched polymer onto the basal plane of GO nanosheets, the feature diffraction peak broadened and left-shifted to $9.18^{\circ}$, corresponding to a $0.963 \mathrm{~nm}$ d-space. Because HBE was a kind of highly branched 3D spherical macromolecule, the introduction of a hyperbranched polymer weakened the interactions and increased the distance between graphene layers. This result was further confirmed by the Raman analysis. According to Figure 5, both GO and GO-HBE-COOH displayed two characteristic broad peaks at $1350 \mathrm{~cm}^{-1}$ (D band) and $1592 \mathrm{~cm}^{-1}$ (G band). D band was assigned to $s p^{3}$ hybridized carbons or the disordered graphite structure, and $\mathrm{G}$ band was attributed to $s p^{2}$ hybridized carbon atoms or the highly oriented graphite structure. Furthermore, the intensity ratio of $\mathrm{D}$ band to $\mathrm{G}$ band $\left(I_{D} / I_{G}\right)$ in GO-HBE-COOH was slightly greater than that of GO, indicating an increase in surface defects after GO was modified with HBPs. It was concluded that HBPs was successfully grafted to the surfaces or edges of GO nanosheets.

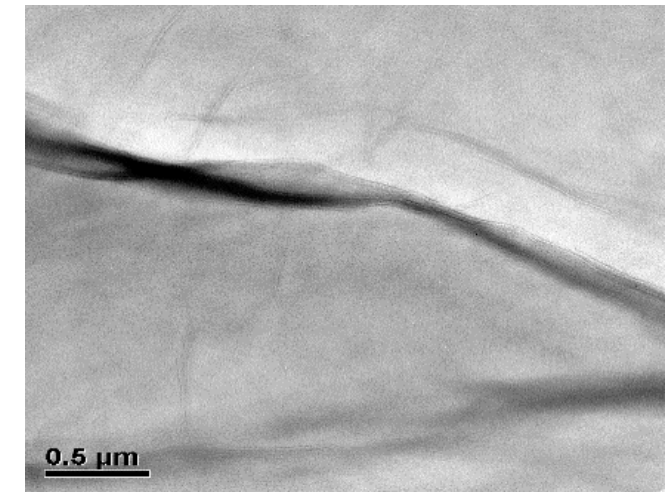

(a)

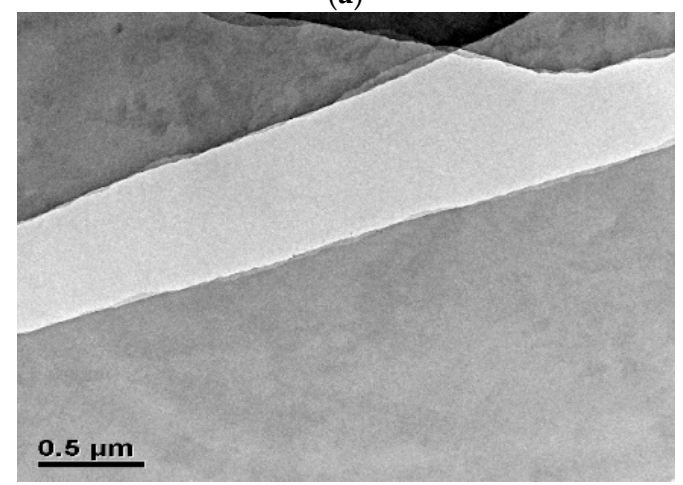

(c)

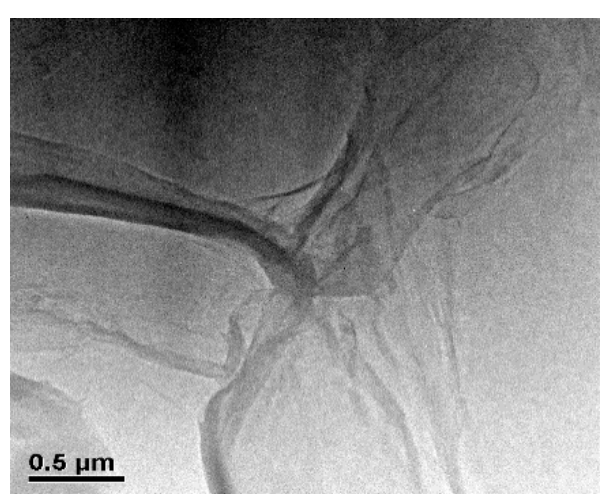

(b)

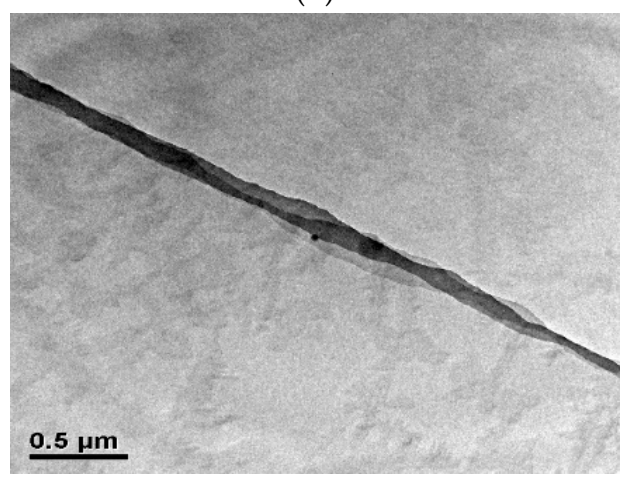

(d)

Figure 3. The TEM images of GO (a,b) and GO-HBE-COOH (c,d).

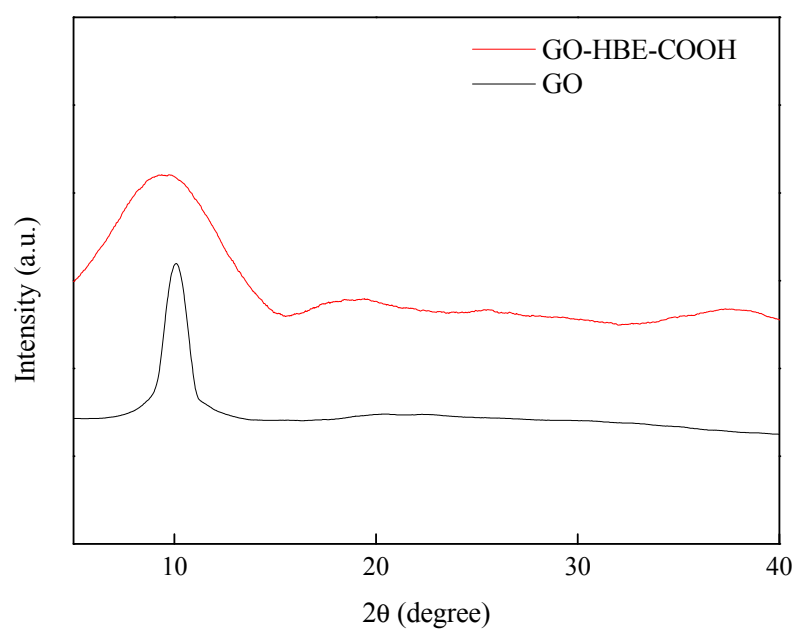

Figure 4. The XRD patterns of GO and GO-HBE-COOH. 


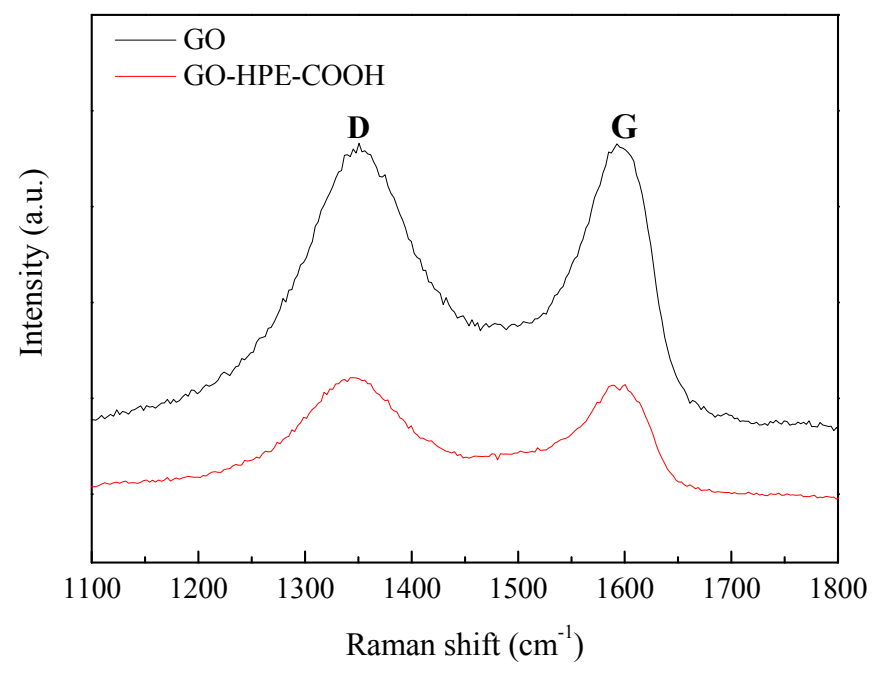

Figure 5. The Raman spectra of GO and GO-HBE-COOH.

\subsection{Characterization of Composite NF Membranes}

In order to elucidate the successful inclusion of GO-HBE-COOH nanosheets into the nanocomposite active layer, ATR-FTIR analysis was used to analyze the chemical groups on the surface of the membrane (Figure 6). The characteristic peaks of TFC-blank and TFN-GHC membranes at 1621, 1585 , and $3410 \mathrm{~cm}^{-1}$ were attributed to the carbonyl stretching vibration of amide-I, the couplings of in-plane $\mathrm{N}-\mathrm{H}$ bending, and the $\mathrm{C}-\mathrm{N}$ stretching vibrations of amide II band, and the hydroxyl stretching vibration, respectively. This confirmed that the formation of PPA active layer on the top of PSU support after IP reaction. Compared to TFC-blank membrane, TFN-GHC membranes presented new absorption peaks at 2930, 2868, and $1724 \mathrm{~cm}^{-1}$, derived from the characteristic peaks of GO-HBE-COOH. Furthermore, these characteristic peaks and the broad band at $3410 \mathrm{~cm}^{-1}$ were enhanced with the increase of GO-HBE-COOH concentration. These results indicated that GO-HBE-COOH nanosheets were successfully embedded into the PPA active layer via IP reaction. Besides the hydrogen bonding between the oxygen-containing groups of $\mathrm{GO}-\mathrm{HBE}-\mathrm{COOH}$ and the PPA polymer, GO-HBE-COOH probably reacted with PIP and TMC monomers due to the abundant carboxyl groups. It was speculated that $\mathrm{GO}-\mathrm{HBE}-\mathrm{COOH}$ nanosheets were introduced into the active layer via covalent bindings (Figure 7), which ensured the long-term stability of GO-HBE-COOH nanosheets in the PPA matrix.

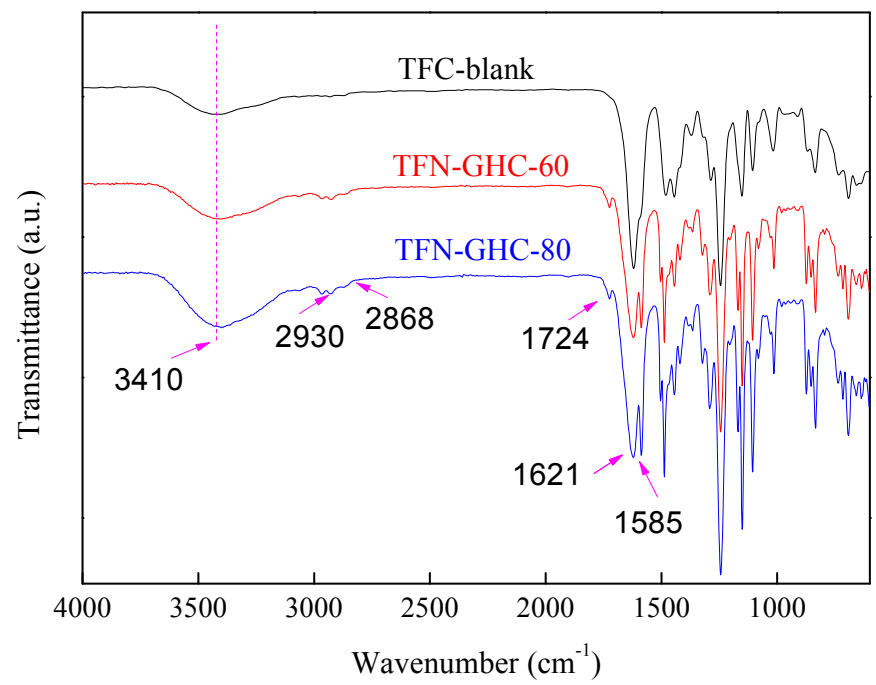

Figure 6. The ATR-FTIR spectra of composite NF membranes. 


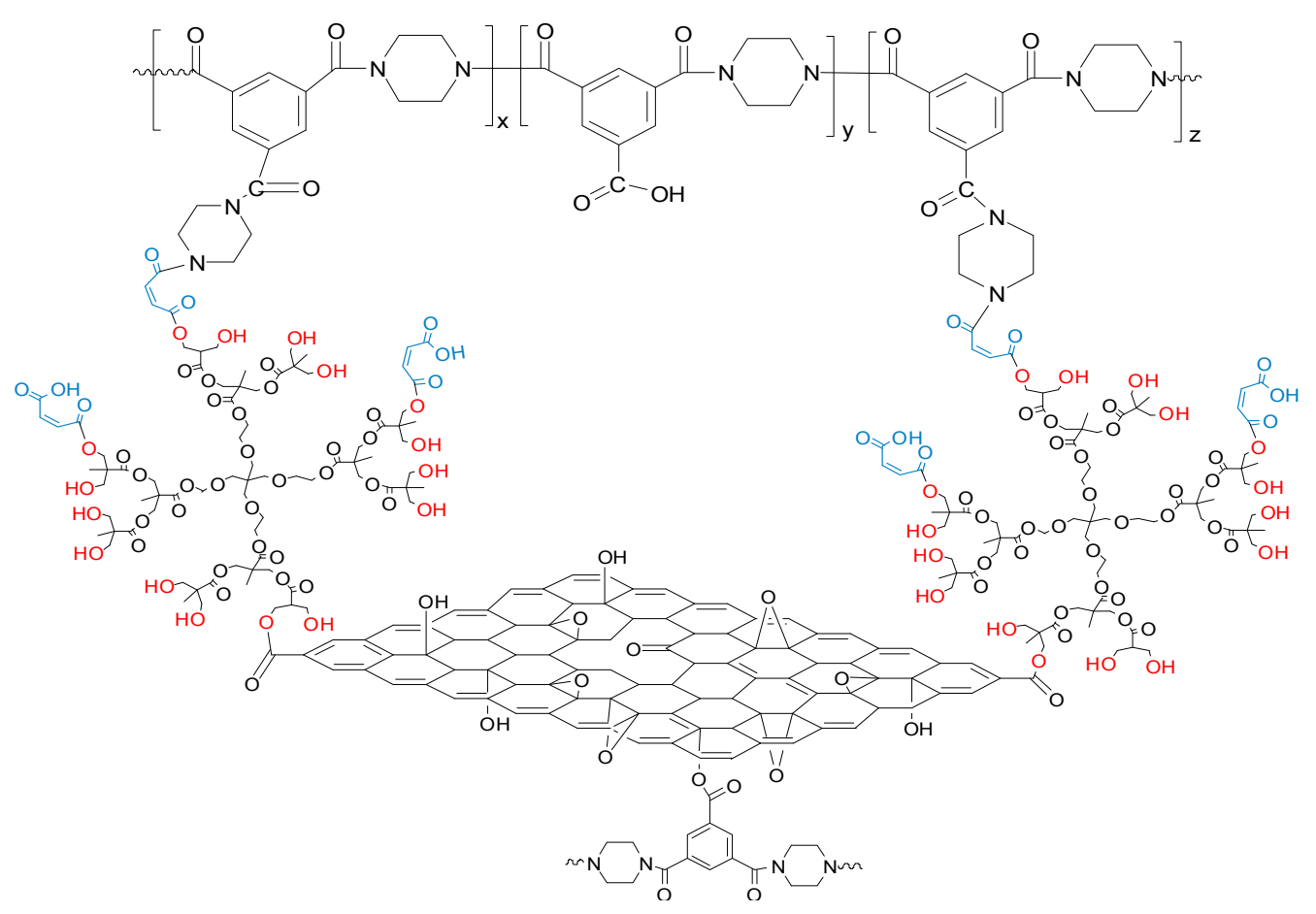

Figure 7. The schematic illustration of interactions between GO-HBE-COOH and PPA.

As shown in Figure 8, all the composite NF membranes exhibited typically composite structures consisting of an ultrathin active layer and a porous sublayer. Beside TFN-GHC-80 membrane, the other membranes exhibited the dense active layers with discrete nodular structures. When the GO-HBE-COOH concentration in the aqueous phase reached $80 \mathrm{ppm}$, some block shapes appeared on the top surface of the active layer which may be caused by the agglomeration of excessive GO-HBE-COOH nanosheets. Interfacial polymerization belongs to a reaction-diffusion process. The diffusion rate of the PIP monomer plays a crucial role in the formation of the PPA active layer. After introducing GO-HBE-COOH into the PIP aqueous phase, the steric hindrance effect arose from GO-HBE-COOH nanosheets and the hydrogen bonding between the PIP monomer and the abundant oxygen-containing hydrophilic groups of GO-HBE-COOH would slow down the PIP diffusion into the organic phase during the IP process [32]. Consequently, the amount of PIP monomers available for the IP reaction decreased within the same reaction time. Correspondingly, the thickness of the nanocomposite active layers obviously reduced with the increase of GO-HBE-COOH concentration. Notably, it seemed that the thickness of the nanocomposite active layer was too small to measure when the GO-HBE-COOH concentration was $80 \mathrm{ppm}$. In addition, the reduction in the thickness of the active layer was expected to reduce the membrane filtration resistance and increase the membrane permeability.

According to Figure 9 and Table 2, the surface roughness of the TFN-GHC membranes firstly decreased and then increased with an increase in the concentration of GO-HBE-COOH as compared with the TFC-blank membrane. As discussed above, the introduction of GO-HBE-COOH nanosheets slowed down the diffusion rate of the PIP monomer to the organic phase and accordingly hindered the formation of the PPA active layer, not only leading to a decrease of the active layer thickness but also to a reduction of the surface roughness. Nevertheless, when the GO-HBE-COOH concentration reached $80 \mathrm{ppm}$, due to the agglomeration of the excessive GO-HBE-COOH nanosheets, some block shapes appeared on the top surface of the active layer, resulting in an increase of the surface roughness. 

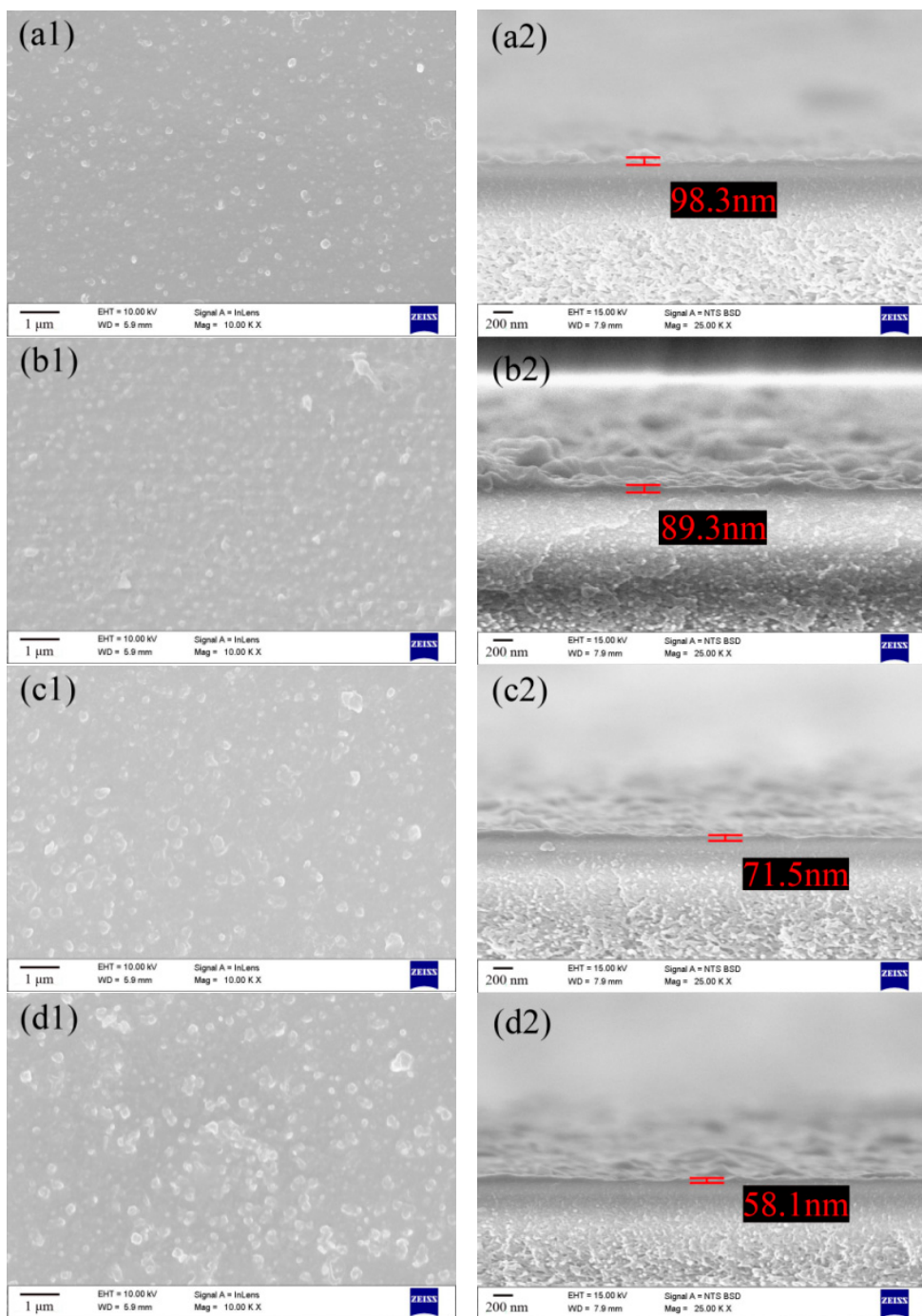

(d2)
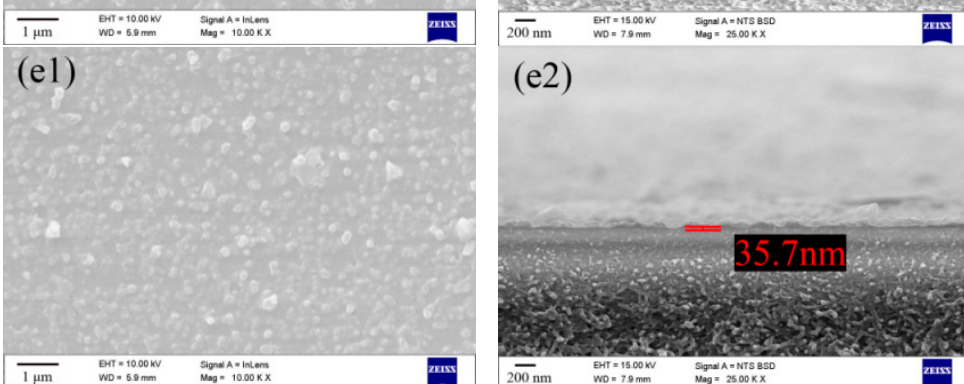

(e2)
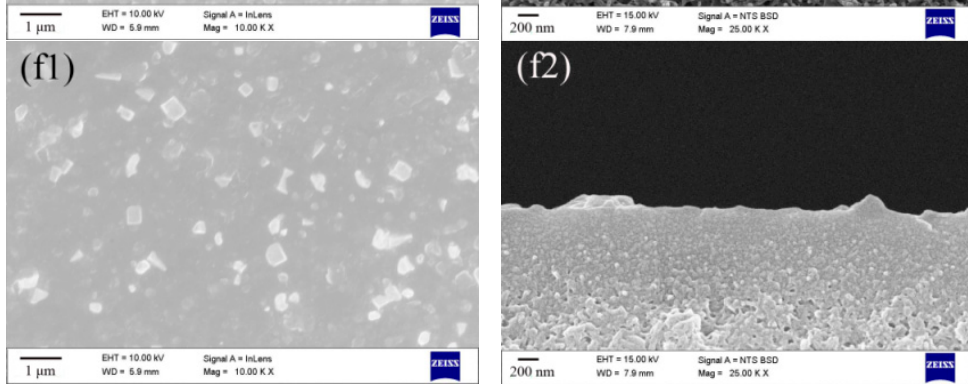

Figure 8. The SEM images of surfaces and cross-sections of composite NF membranes: TFC-blank (a1,a2), TFN-GHC-10 (b1,b2), TFN-GHC-20 (c1,c2), TFN-GHC-40 (d1,d2), TFN-GHC-60 (e1,e2), TFN-GHC-80 (f1,f2). 

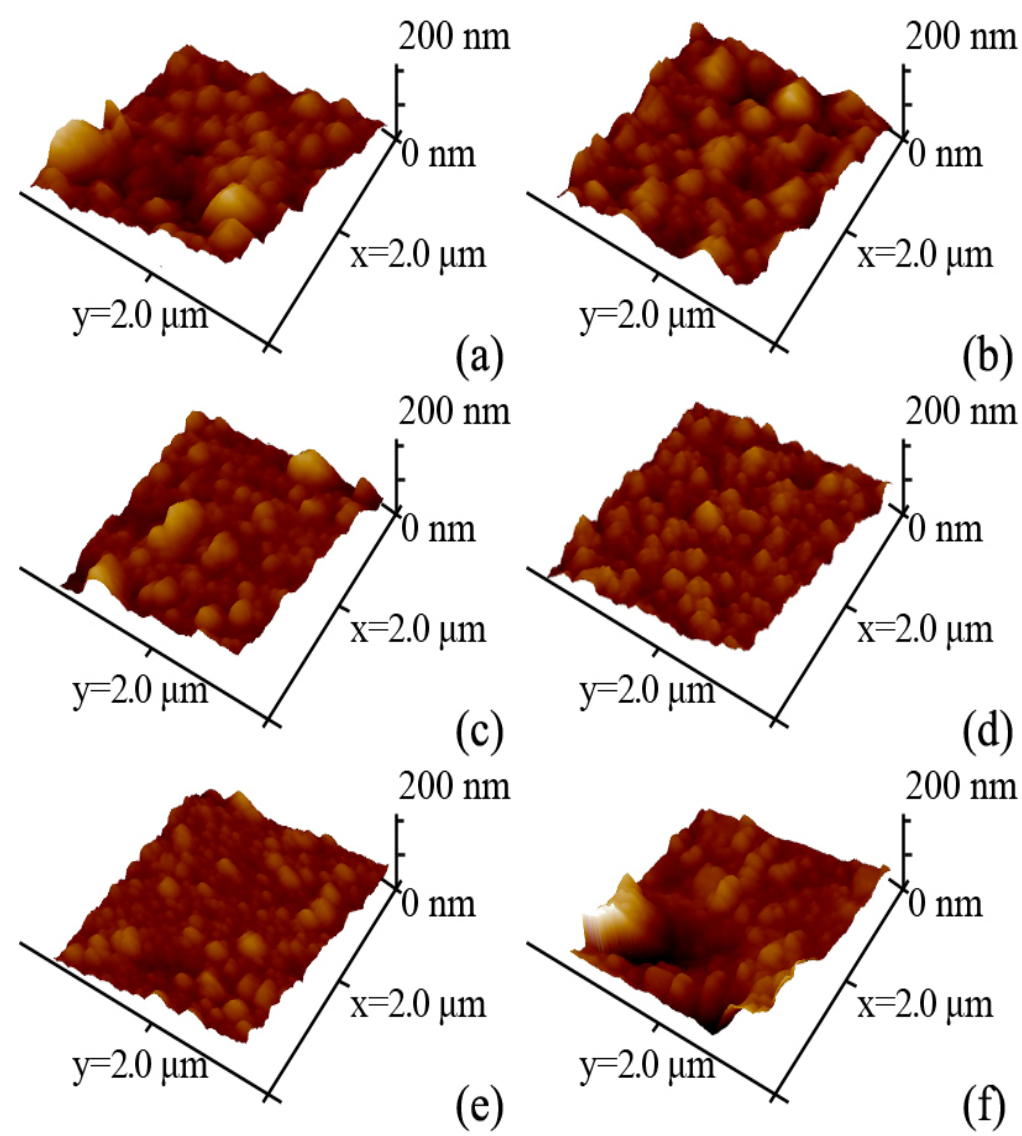

Figure 9. The three-dimensional AFM surface topography images of composite NF membranes: (a) TFC-blank; (b) TFN-GHC-10; (c) TFN-GHC-20; (d) TFN-GHC-40; (e) TFN-GHC-60; (f) TFN-GHC-80.

Table 2. The roughness of composite NF membranes.

\begin{tabular}{cccc}
\hline \multirow{2}{*}{ Membrane ID } & \multicolumn{3}{c}{ Roughness } \\
\cline { 2 - 4 } & $\boldsymbol{R} \boldsymbol{a}(\mathbf{n m})$ & $\boldsymbol{R} \boldsymbol{q}(\mathbf{n m})$ & $\boldsymbol{R} \boldsymbol{z}(\mathbf{n m})$ \\
\hline TFC-blank & 23.2 & 30.9 & 90.9 \\
TFN-GHC-10 & 21.9 & 28.0 & 83.1 \\
TFN-GHC-20 & 19.5 & 26.6 & 91.2 \\
TFN-GHC-40 & 16.0 & 20.0 & 69.4 \\
TFN-GHC-60 & 13.8 & 17.9 & 54.1 \\
TFN-GHC-80 & 26.7 & 39.8 & 93.5 \\
\hline
\end{tabular}

The water contact angle (WCA) of non-porous membranes such as NF and RO is mainly affected by the intrinsic wettability of the membrane material and the surface roughness. In general, the non-porous membrane having a rougher surface with the stronger hydrophilicity displays a smaller WCA. In this study, the variation of surface roughness was within $22 \mathrm{~nm}$, so the influence of surface roughness on WCA could be negligible. As shown in Figure 10, the WCA of TFN-GHC membranes gradually decreased with the increase of GO-HBE-COOH concentration. This meant that TFN-GHC NF membranes became more and more hydrophilic with the increasing GO-HBE-COOH concentration. It was well understood that the enhanced hydrophilicity of TFN-GHC membranes was ascribed to the incorporated GO-HBE-COOH nanosheets having a large number of hydrophilic oxygen-containing groups, particularly an increasing amount of carboxyl groups. 


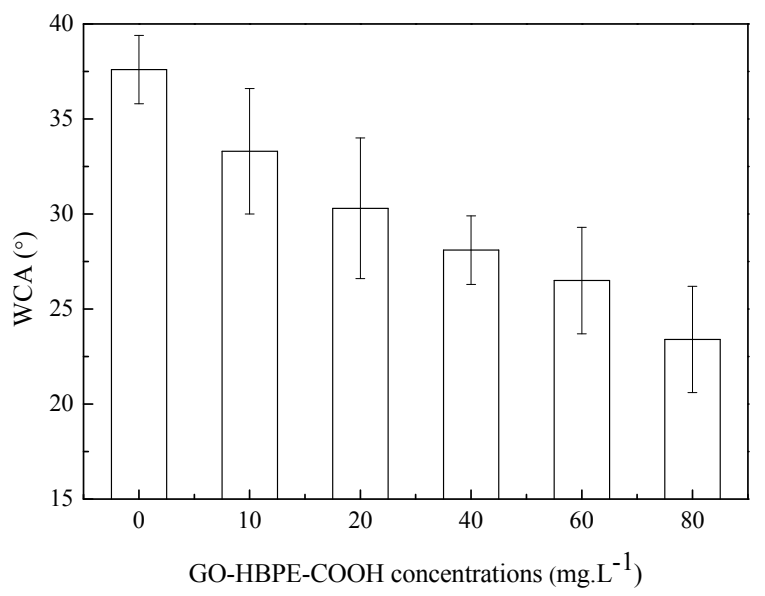

Figure 10. The effect of the GO-HBE-COOH concentration on WCA.

\subsection{Membrane Performance}

The effects of the GO-HBE-COOH concentration on the performance of composite NF membranes were evaluated according to the water flux and salt rejection (Figure 11). As discussed above, the nanocomposite active layer became thinner, smoother, and more hydrophilic with the addition of 10-60 ppm GO-HBE-COOH. As a result, the water fluxes of the corresponding TFN-GHC membranes gradually increased due to the reduction of membrane filtration resistance and a faster water flow on the membrane surface with less friction [30]. Additionally, the GO-HBE-COOH nanofiller having 2D nanosheets and a 3D spherical structure could increase the free volume between polymer chains and provided the additional water channels due to the nanometer-sized voids inside HBPs [20,33]. When the GO-HBE-COOH concentration reached the critical value of $60 \mathrm{ppm}$, TFN-GHC-60 membrane showed the maximum water flux of $48.0 \mathrm{~L} \cdot \mathrm{m}^{-2} \cdot \mathrm{h}^{-1}$ equal to $143.7 \%$ of the TFC-blank membrane. More importantly, the TFN-GHC-60 NF membranes exhibited a high enhancement in water permeability while maintaining high rejection of salts, indicating that the introduction of an appropriate amount of GO-HBE-COOH nanosheets into the PPA active layer did not generate membrane defects. However, the water flux of TFN-GHC- 80 membrane turned to decrease at the high concentration of $80 \mathrm{ppm}$ because of the agglomeration of excessive GO-HBE-COOH nanofillers. Although the introduction of GO-HBE-COOH nanofillers in the aqueous phase resulted in the formation of a looser nanocomposite active layer which was adverse to the salt rejection, a large amount of ionizable and negatively charged groups in GO-HBE-COOH contributed to reducing the passage of salt due to the electrostatic repulsion effect. Consequently, TFN-GHC NF membranes retained a high salt rejection similar to the TFC-blank membrane.

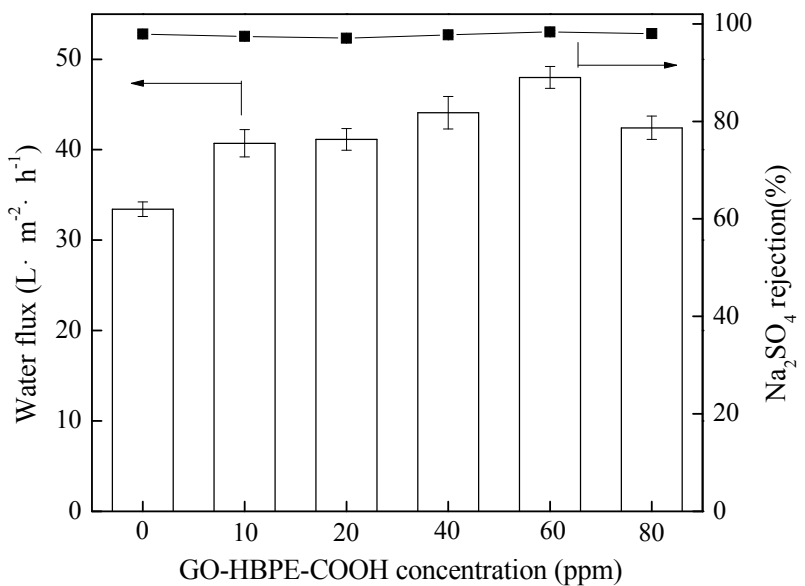

Figure 11. The water fluxes and rejections of composite NF membranes. 
It is known that the rejection of a charged NF membrane to an electrolyte is not only determined by the steric effect, but also depends on the electrostatic repulsion effect between the membrane and electrolyte. As shown in Figure 12, the salt rejections followed the order of $\mathrm{Na}_{2} \mathrm{SO}_{4}>\mathrm{MgSO}_{4}>\mathrm{MgCl}_{2}>$ $\mathrm{NaCl}$, which was consistent with the separation characteristics of a negatively charged NF membrane. It was revealed that the co-ion played an important role in the rejection and transport rather than the counter-ion [34]. The negatively charged NF membrane would generate a stronger repulsive force to a divalent co-ion $\left(\mathrm{SO}_{4}{ }^{2-}\right)$ than to a monovalent co-ion $\left(\mathrm{Cl}^{-}\right)$due to the electrostatic repulsion effect. Furthermore, the $\mathrm{SO}_{4}{ }^{2-}$ ion exhibits a lower diffusion coefficient and a larger Stokes radius than $\mathrm{Cl}^{-}$ ion (Table 1). Therefore, it was more difficult for the $\mathrm{SO}_{4}{ }^{2-}$ ion to pass through the active layer in comparison with $\mathrm{Cl}^{-}$ion. Meanwhile, the negatively charged NF membrane had a stronger attraction for a divalent counter-ion $\left(\mathrm{Mg}^{2+}\right)$ than a monovalent counter-ion $\left(\mathrm{Na}^{+}\right)$. Consequently, the negatively charged NF membrane favored the rejection of the $\mathrm{Na}^{+}$ion rather than the $\mathrm{Mg}^{2+}$ ion, resulting in a higher rejection of $\mathrm{Na}_{2} \mathrm{SO}_{4}$ than $\mathrm{MgSO}_{4}$. For the salt rejection having the same monovalent co-ion $\left(\mathrm{Cl}^{-}\right)$, the higher rejection to $\mathrm{MgCl}_{2}$ than to $\mathrm{NaCl}$ was presumably due to the larger steric hindrance of the $\mathrm{Mg}^{2+}$ ion than to the $\mathrm{Na}^{+}$ion.

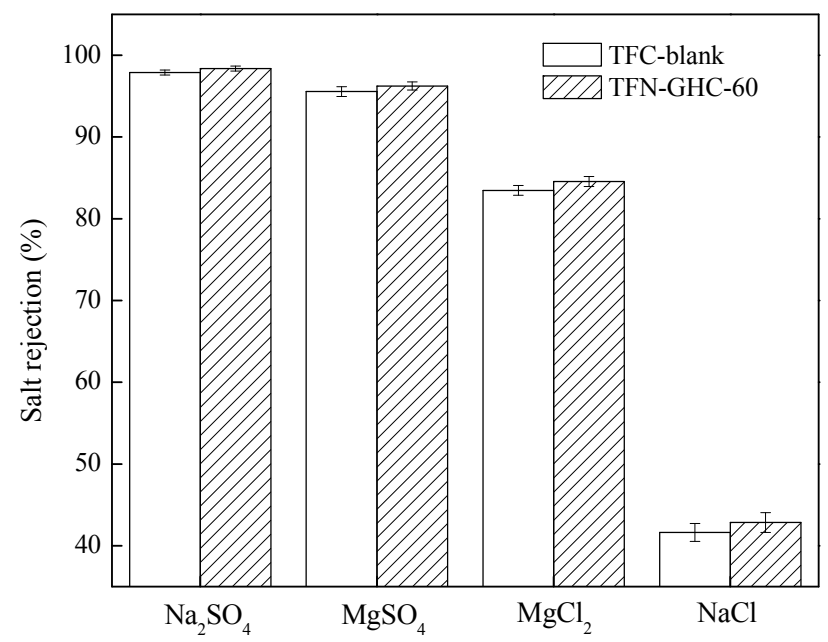

Figure 12. The four kinds of salt rejections of composite NF membranes.

Fouling tests were conducted on the TFC-blank, TFN-GHC-60, and TFN-GHC-80 membranes using BSA as the protein foulant. As shown in Figure 13, compared with the TFC-blank membrane, both the TFN-GHC NF membrane displayed a slower normalized flux decline, and the normalized flux decreased slowly with the increase of GO-HBE-COOH concentration. At the end of the second cycle of BSA filtration, the normalized flux of TFN-GHC-80 and TFN-GHC-60 membrane decreased by $7.0 \%$ and $12.0 \%$, respectively, which was remarkably less than that of the TFC-blank membrane $(30.0 \%)$. These results indicated that the TFN-GHC NF membranes were more resistant to protein contamination after the addition of GO-HBE-COOH nanosheets to the active layer. The antifouling abilities are typically affected by many membrane characteristics including hydrophilicity, surface roughness, and surface charge. Generally, a smooth membrane surface having a relatively strong hydrophilicity and a high surface charge exhibits a better antifouling ability. After the covalent binding of GO-HBE-COOH in the top active layer, TFN-GHC NF membranes showed a smoother surface with the enhancement in the membrane hydrophilicity and surface charge density, which promoted the formation of a regular hydration layer on the hydrophilic surface via hydrogen bonding, as well as strengthening the electrostatic repulsion between the negatively charged membrane surface and BSA. This would greatly reduce the BSA adsorption on the nanocomposite surface of the TFN-GHC NF membranes. 


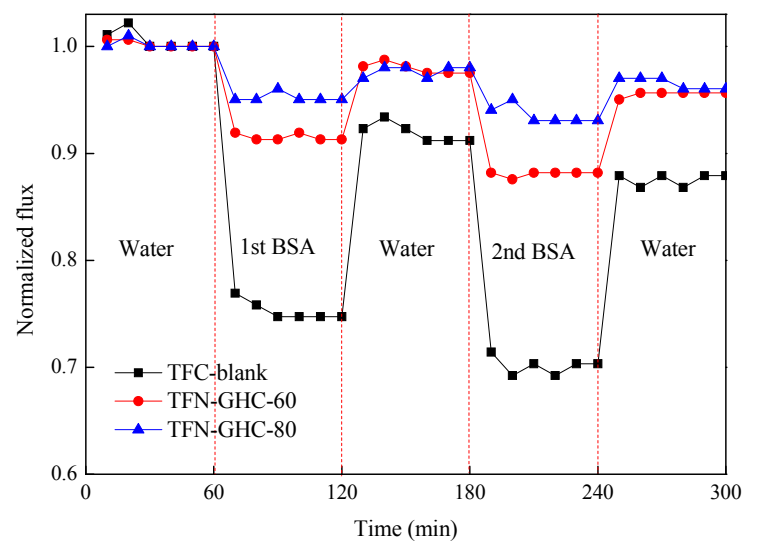

Figure 13. The time-dependent normalized flux of composite NF membranes.

In view of the chlorinated degradation mechanism, the degradation of polyamide membranes normally occurs via a nucleophilic substitution reaction between chlorine and the hydrogen of the secondary amide group ( $-\mathrm{NH})$ [35]. So the secondary amines of PIP reacted with TMC forms the tertiary amines, which enhances the chlorine tolerance of PPA membrane. However, chlorinated degradation still occurred at the low abundance noncross-linked nitrogen atoms of PPA membrane [36]. To assess the chlorine resistance of TFC-blank and TFN-GHC NF membranes, the permeaselectivity was measured before and after the chlorine treatment. As shown in Figures 14 and 15, all composite NF membranes displayed a reduction in salt rejection and an enlargement in water flux after chlorination, but the TFN-GHC membranes embedded with GO-HBE-COOH into the active layer showed an obvious inhibiting effect in water flux and salt rejection variations. After $72 \mathrm{~h}$ of chlorination exposure, the normalized salt rejections of the TFN-GHC- 80 and TFN-GHC-60 membranes reduced to $81.8 \%$ and $72.0 \%$, respectively, but the TFC-blank membrane reduced to $62.2 \%$, which indicated that the chlorine resistance of the TFN-GHC NF membranes was enhanced with the increasing GO-HBE-COOH concentration. It was found that GO in the polymer matrix could trap the radicals because they had phenolic moieties with the radical scavenging ability [37]. Therefore, GO could absorb the chlorine radical to form $\mathrm{O}-\mathrm{Cl}$ leading to a decrease in chlorine radical attacking the polyamide layer [38]. Because GO-HBE-COOH kept the skeleton structure of GO after modification with the hyperbranched polymer, it was deduced that GO-HBE-COOH could increase the chlorine resistance of the PPA-based membranes due to the formation of $\mathrm{O}-\mathrm{Cl}$ during the chlorine radical attack. In addition, GO-HBE-COOH provided additional protections due to the huge specific surface area and the hydrogen bonding between PPA and GO-HBE-COOH [39].

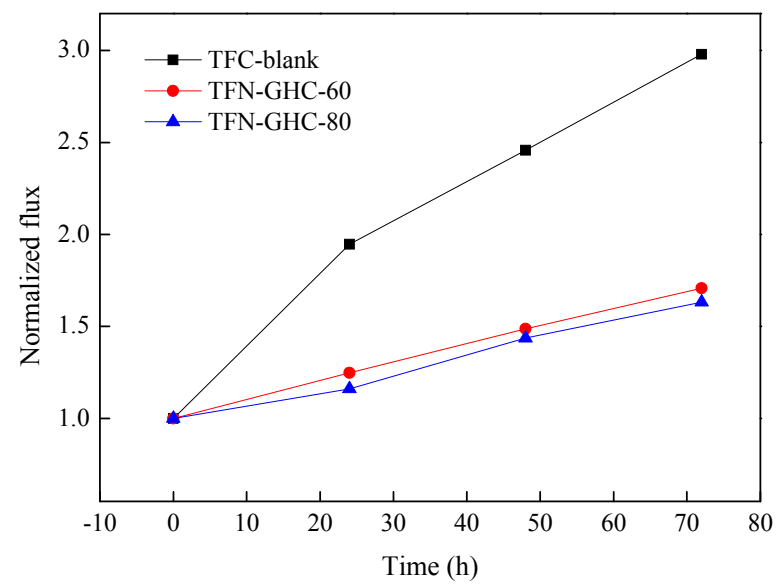

Figure 14. The effect of the chlorination exposure time on the water fluxes of composite NF membranes. 


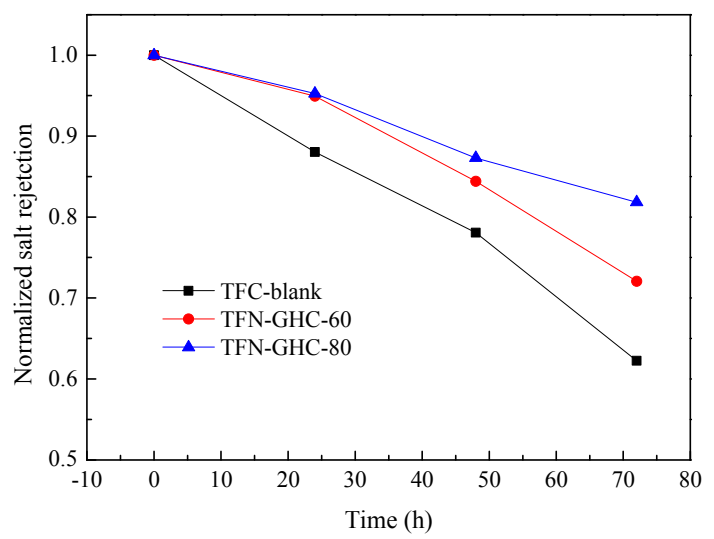

Figure 15. The effect of the chlorination exposure time on the salt rejections of composite NF membranes.

\subsection{Mechanisms}

It was well known that the growth rate of the polyamide layer is limited by the diffusion of the aqueous-phase monomer through the newly-formed polyamide [40]. That is, the diffusivity of amine monomer during the IP reaction significantly affects the properties, and performance of the fabricated TFC membrane. In this study, using the porous PSU supports, the introduction of GO-HBE-COOH nanosheets into the PIP aqueous phase could effectively hinder the diffusion rate of PIP monomers into the organic phase due to the steric hindrance effect of GO-HBE-COOH nanosheets and the hydrogen bonding between the PIP molecules and the oxygen-containing hydrophilic groups of GO-HBE-COOH nanosheets. At the same time, the synergistic combination of 3D hyperbranched macromolecules in GO-HBE-COOH nanosheets increased the free volume between the PPA chains and provided additional channels. Therefore, in comparison with the TFC-blank membrane, the TFN-GHC NF membrane exhibited a thinner, looser, and smoother nanocomposite active layer which resulted in reducing the membrane filtration resistance and obviously increasing the membrane permeability. Furthermore, the TFN-GHC NF membrane showed an enhanced hydrophilic and negatively charged surface due to a large number of hydrophilic and ionizable groups from GO-HBE-COOH nanosheets. As a result, TFN-GHC NF membranes embedded with GO-HBE-COOH nanosheets demonstrated a superior permeaselectivity, antifouling ability, and chlorine resistance over the traditional PPA-TFC NF membranes (Figure 16).

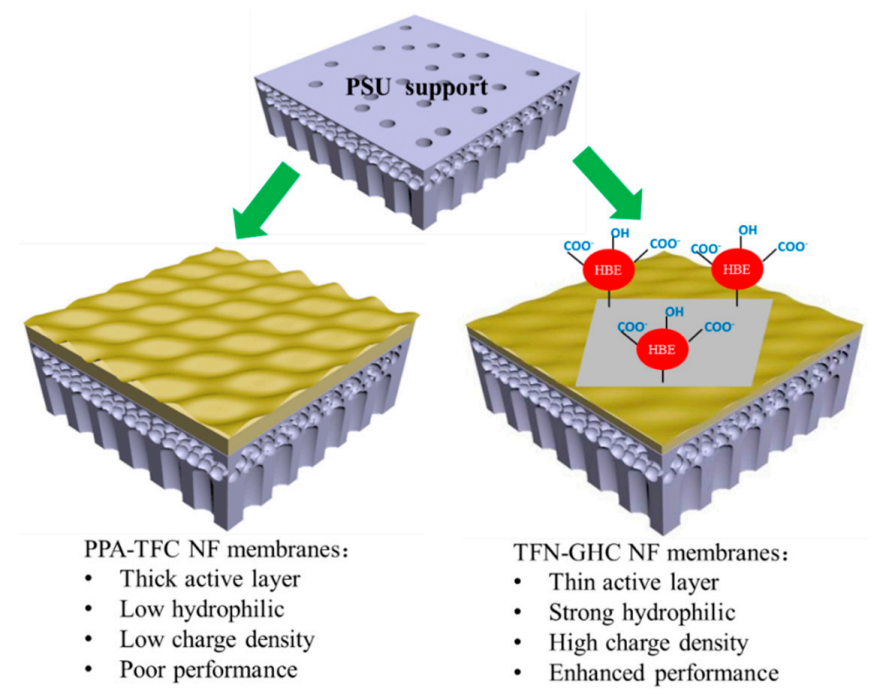

Figure 16. The schematic representation of speculated active layer formation. 


\section{Conclusions}

GO was firstly modified by 3D hyperbranched polyester and then carboxylated by succinic anhydride, and the resultant GO-HBE-COOH nanofiller was successfully incorporated into the PPA active layer via the IP process. The influences of the GO-HBE-COOH concentration on the morphologies, properties, and performance of the fabricated TFN NF membranes were investigated. With the addition of $60 \mathrm{ppm}$ GO-HBE-COOH in the aqueous phase, the TFN-GHC-60 NF membrane exhibited the optimal water flux without a sacrifice of the salt rejection. Such a significant increase of the water flux resulted from the formation of a thinner, looser, and smoother nanocomposite active layer with more hydrophilicity, which was ascribed to the diffusion rate of PIP monomers hindered by the introduction of GO-HBE-COOH nanosheets. Furthermore, TFN-GHC NF membranes demonstrated a better antifouling ability and chlorine resistance with the increase of GO-HBE-COOH concentration. It was found that the high increase of antifouling performance was attributed to the smoother surface with the enhancement in the hydrophilicity and the negative charge, and the improved chlorine resistance was due to the protection of the PPA active layer provided by GO-HBE-COOH nanosheets. Therefore, we could use GO-HBE-COOH as a novel nanofiller incorporated into the active layer to develop the high-performance TFN NF membrane.

Author Contributions: Conceived and designed the experiments, Q.X. and W.S.; Investigation, S.Z. and Q.X.; Data Curation, S.Z. and H.M; Writing-Review \& Editing, Q.X. and X.G; Supervision, Z.H. and Q.X.; Funding Acquisition, Q.X., Z.H. and W.S.

Funding: This research was funded by the Science \& Technology Planning Project of Xiamen City, China (No. 3502Z20172008), the Scientific Research Foundation of Third Institute of Oceanography, SOA (No. 2016036), Xiamen Southern Oceanographic Center (16PFW008SF15), the Fundamental Research Funds for the Central Universities (No. 20720170027) and the National Natural Science Foundation of China (No. 21736009).

Acknowledgments: The authors thank Qiaorong Han (School of Chemistry and Materials Science, Nanjing Normal University) and Birong Zeng (Department of Materials Science and Engineering, College of Materials, Xiamen University) for their suggestions about the synthesis and characterization of GO-HPE-COOH.

Conflicts of Interest: The authors declare no conflicts of interest.

\section{References}

1. Mohammad, A.W.; Teow, Y.H.; Ang, W.L.; Chung, Y.T.; Oatley-Radcliffe, D.L.; Hilal, N. Nanofiltration membranes review: Recent advances and future prospects. Desalination 2015, 356, 226-254. [CrossRef]

2. Xu, G.-R.; Xu, J.-M.; Feng, H.-J.; Zhao, H.-L.; Wu, S.-B. Tailoring structures and performance of polyamide thin film composite (PA-TFC) desalination membranes via sublayers adjustment-A review. Desalination 2017, 417, 19-35. [CrossRef]

3. Lau, W.J.; Gray, S.; Matsuura, T.; Emadzadeh, D.; Chen, J.P.; Ismail, A.F. A review on polyamide thin film nanocomposite (TFN) membranes: History, applications, challenges and approaches. Water Res. 2015, 80, 306-324. [CrossRef] [PubMed]

4. Ismail, A.F.; Padaki, M.; Hilal, N.; Matsuura, T.; Lau, W.J. Thin film composite membrane-Recent development and future potential. Desalination 2015, 356, 140-148. [CrossRef]

5. Yin, J.; Zhu, G.C.; Deng, B.L. Graphene oxide (GO) enhanced polyamide (PA) thin-film nanocomposite (TFN) membrane for water purification. Desalination 2016, 379, 93-101. [CrossRef]

6. Lai, G.S.; Lau, W.J.; Goh, P.S.; Ismail, A.F.; Yusof, N.; Tan, Y.H. Graphene oxide incorporated thin film nanocomposite nanofiltration membrane for enhanced salt removal performance. Desalination 2016, 387, 14-24. [CrossRef]

7. Wang, J.; Zhao, C.W.; Wang, T.; Wu, Z.; Li, X.; Li, J.S. Graphene oxide polypiperazine-amide nanofiltration membrane for improving flux and anti-fouling in water purification. RSC Adv. 2016, 6, 82174-82185. [CrossRef]

8. Bano, S.; Mahmood, A.; Kim, S.J.; Lee, K.H. Graphene oxide modified polyamide nanofiltration membrane with improved flux and antifouling properties. J. Mater. Chem. A 2015, 3, 2065-2071. [CrossRef] 
9. Xia, S.J.; Yao, L.J.; Zhao, Y.; Li, N.N.; Zheng, Y. Preparation of graphene oxide modified polyamide thin film composite membranes with improved hydrophilicity for natural organic matter removal. Chem. Eng. J. 2015, 280, 720-727. [CrossRef]

10. Ali, M.E.A.; Wang, L.Y.; Wang, X.Y.; Feng, X.S. Thin film composite membranes embedded with graphene oxide for water desalination. Desalination 2016, 386, 67-76. [CrossRef]

11. Kim, F.; Cote, L.J.; Huang, J. Graphene oxide: Surface activity and two-dimensional assembly. Adv. Mater. 2010, 22, 1954-1958. [CrossRef] [PubMed]

12. Cote, L.J.; Kim, J.; Tung, V.C.; Luo, J.Y.; Kim, F.; Huang, J.X. Graphene oxide as surfactant sheets. Pure Appl. Chem. 2011, 83, 95-110. [CrossRef]

13. Hung, W.S.; An, Q.F.; De Guzman, M.; Lin, H.Y.; Huang, S.H.; Liu, W.R.; Hu, C.C.; Lee, K.R.; Lai, J.Y. Pressure-assisted self-assembly technique for fabricating composite membranes consisting of highly ordered selective laminate layers of amphiphilic graphene oxide. Carbon 2014, 68, 670-677. [CrossRef]

14. Xie, Q.L.; Shao, W.Y.; Zhang, S.S.; Hong, Z.; Wang, Q.Q.; Zeng, B.R. Enhancing the performance of thin-film nanocomposite nanofiltration membranes using MAH-modified GO nanosheets. RSC Adv. 2017, 7, 54898-54910. [CrossRef]

15. Zheng, Y.C.; Li, S.P.; Weng, Z.L.; Gao, C. Hyperbranched polymers: Advances from synthesis to applications. Chem. Soc. Rev. 2015, 44, 4091-4130. [CrossRef] [PubMed]

16. Gao, C.; Yan, D. Hyperbranched polymers: From synthesis to applications. Prog. Polym. Sci. 2004, 29, 183-275. [CrossRef]

17. Qiu, Z.L.; Kong, X.; Yuan, J.J.; Shen, Y.J.; Zhu, B.K.; Zhu, L.P.; Yao, Z.K.; Tang, C.Y. Cross-linked PVC/hyperbranched polyester composite hollow fiber membranes for dye removal. React. Funct. Polym. 2018, 122, 51-59. [CrossRef]

18. Schulze, A.; Went, M.; Prager, A. Membrane functionalization with hyperbranched polymers. Materials 2016, 9, 706. [CrossRef] [PubMed]

19. Wei, X.Z.; Ong, X.; Yang, J.; Zhang, G.L.; Chen, J.Y.; Wang, J.D. Structure influence of hyperbranched polyester on structure and properties of synthesized nanofiltration membranes. J. Membr. Sci. 2013, 440, 67-76. [CrossRef]

20. Kong, X.; Zhang, Y.; Zeng, S.Y.; Zhu, B.K.; Zhu, L.P.; Fang, L.F.; Matsuyama, H. Incorporating hyperbranched polyester into cross-linked polyamide layer to enhance both permeability and selectivity of nanofiltration membrane. J. Membr. Sci. 2016, 518, 141-149. [CrossRef]

21. Wei, X.Z.; Yang, J.; Zhang, G.L. Preparation and characterization of nanofiltration membranes synthesized by hyperbranched polyester and terephthaloyl chloride (TPC). Polym. Polym. Compos. 2012, 20, 261-270. [CrossRef]

22. Wei, X.Z.; Zhu, L.P.; Deng, H.Y.; Xu, Y.Y.; Zhu, B.K.; Huang, Z.M. New type of nanofiltration membrane based on crosslinked hyperbranched polymers. J. Membr. Sci. 2008, 323, 278-287. [CrossRef]

23. Wei, X.Z.; Yang, J.; Xu, Y.Y.; Zhu, B.K.; Zhang, G.L. Preparation and characterization of low-pressure nanofiltration membranes and the application in the separation process of dye molecules. J. Porous Mater. 2012, 19, 721-731. [CrossRef]

24. Zhao, F.Y.; Mi, Y.F.; An, Q.F.; Gao, C.J. Preparation and applications of positively charged polyethyleneimine nanofiltration membrane. Prog. Chem. 2016, 28, 541-551.

25. Chiang, Y.C.; Hsub, Y.Z.; Ruaan, R.C.; Chuang, C.J.; Tung, K.L. Nanofiltration membranes synthesized from hyperbranched polyethyleneimine. J. Membr. Sci. 2009, 326, 19-26. [CrossRef]

26. Wei, X.Z.; Hong, J.L.; Zhu, S.S.; Chen, J.Y.; Lv, B.S. Structure-performance study of polyamide composite nanofiltration membranes prepared with polyethyleneimine. J. Mater. Sci. 2017, 52, 11701-11714. [CrossRef]

27. Sun, S.P.; Hatton, T.A.; Chung, T.S. Hyperbranched polyethyleneimine induced cross-linking of polyamide-imide nanofiltration hollow fiber membranes for effective removal of ciprofloxacin. Environ. Sci. Technol. 2011, 45, 4003-4009. [CrossRef] [PubMed]

28. Misdan, N.; Lau, W.J.; Ismail, A.F.; Matsuura, T. Formation of thin film composite nanofiltration membrane: Effect of polysulfone substrate characteristics. Desalination 2013, 329, 9-18. [CrossRef]

29. Wang, D.X.; Su, M.; Yu, Z.Y.; Wang, X.L.; Ando, M.; Shintani, T. Separation performance of a nanofiltration membrane influenced by species and concentration of ions. Desalination 2005, 175, 219-225. [CrossRef] 
30. Xie, Q.L.; Zhang, S.S.; Xiao, Z.Y.; Hu, X.F.; Hong, Z.; Yi, R.Z.; Shao, W.Y.; Wang, Q.Q. Preparation and characterization of novel alkali-resistant nanofiltration membranes with enhanced permeation and antifouling properties: The effects of functionalized graphene nanosheets. RSC Adv. 2017, 7, 18755-18764. [CrossRef]

31. Wu, H.Q.; Tang, B.B.; Wu, P.Y. Development of novel $\mathrm{SiO}_{2}-\mathrm{GO}$ nanohybrid/polysulfone membrane with enhanced performance. J. Membr. Sci. 2014, 451, 94-102. [CrossRef]

32. Chae, H.R.; Lee, J.; Lee, C.H.; Kim, I.C.; Park, P.K. Graphene oxide-embedded thin-film composite reverse osmosis membrane with high flux, anti-biofouling, and chlorine resistance. J. Membr. Sci. 2015, 483, 128-135. [CrossRef]

33. Li, Y.; He, G.; Wang, S.; Yu, S.; Pan, F.; Wu, H.; Jiang, Z. Recent advances in the fabrication of advanced composite membranes. J. Mater. Chem. A 2013, 1, 10058-10077. [CrossRef]

34. Garcia-Aleman, J.; Dickson, J.M. Permeation of mixed-salt solutions with commercial and pore-filled nanofiltration membranes: Membrane charge inversion phenomena. J. Membr. Sci. 2004, 239, 163-172. [CrossRef]

35. Glater, J.; Hong, S.K.; Elimelech, M. The search for a chlorine-resistant reverse-osmosis membrane. Desalination 1994, 95, 325-345. [CrossRef]

36. Tang, Y.J.; Xu, Z.L.; Xue, S.M.; Wei, Y.M.; Yang, H. A chlorine-tolerant nanofiltration membrane prepared by the mixed diamine monomers of PIP and BHTTM. J. Membr. Sci. 2016, 498, 374-384. [CrossRef]

37. Yuan, B.H.; Bao, C.L.; Song, L.; Hong, N.N.; Liew, K.M.; Hu, Y. Preparation of functionalized graphene oxide/polypropylene nanocomposite with significantly improved thermal stability and studies on the crystallization behavior and mechanical properties. Chem. Eng. J. 2014, 237, 411-420. [CrossRef]

38. Shao, F.F.; Dong, L.F.; Dong, H.Z.; Zhang, Q.; Zhao, M.; Yu, L.Y.; Pang, B.L.; Chen, Y.J. Graphene oxide modified polyamide reverse osmosis membranes with enhanced chlorine resistance. J. Membr. Sci. 2017, 525, 9-17. [CrossRef]

39. Choi, W.; Choi, J.; Bang, J.; Lee, J.H. Layer-by-layer assembly of graphene oxide nanosheets on polyamide membranes for durable reverse-osmosis applications. ACS Appl. Mater. Interfaces 2013, 5, 12510-12519. [CrossRef] [PubMed]

40. Matthews, T.D.; Yan, H.; Cahill, D.G.; Coronell, O.; Mariñas, B.J. Growth dynamics of interfacially polymerized polyamide layers by diffuse reflectance spectroscopy and rutherford backscattering spectrometry. J. Membr. Sci. 2013, 429, 71-80. [CrossRef] 